\title{
Comments on "The Intensification of the Low-Level Jet during the Development of Mesoscale Convective Systems on a Mei-Yu Front"
}

\author{
JOHN W. NIELSEN-GAMMON \\ Department of Meteorology, Texas A\&M University, College Station, Texas \\ DAVID M. SCHULTZ \\ National Severe Storms Laboratory, Norman, Oklahoma
}

26 August 1998 and 8 March 1999

\section{Introduction}

The recent article by Chen et al. (1998; henceforth Chen) concludes that three processes were important to the development of a particular low-level jet (LLJ) associated with a mei-yu front: (1) a transverse circulation caused by thermal wind adjustment at the entrance region of a jet streak; (2) a direct circulation driven by deep convection along the frontal zone; and (3) slantwise convection maintained by conditional symmetric instability. We find problems with the methodology and interpretation of all three phenomena.

\section{Ageostrophic circulations forced by upper-tropospheric jet dynamics}

Chen (p. 357) argues that the upward branch of a transverse ageostrophic circulation initiates the convection. This ageostrophic circulation is attributed to jet entrance dynamics, based on the existence of a southwesterly jet entrance region at $13 \mathrm{~km}$ (approximately $200 \mathrm{hPa}$ ) and the presence of strong ageostrophic winds in the core of the jet in the cross section (see Chen's Figs. 8-10). Chen's Fig. 8a, however, shows that these ageostrophic winds are not part of a transverse "circulation." In Chen's model simulation, only the southeasterly winds aloft are present. The downward branch, the return northwesterly branch at low levels, and the upward branch are either nonexistent or at least an order of magnitude too weak to complete the hypothesized circulation cell.

To address these issues, we use the NCEP-NCAR reanalysis data (Kalnay et al. 1996). The analyses, while

Corresponding author address: John W. Nielsen-Gammon, Dept. of Meteorology, MS 3150, Texas A\&M University, College Station, TX 77843-3150.

E-mail: n-g@tamu.edu coarse, do reproduce the upper-level trough, jet streak, and ageostrophic winds found in the model simulation by Chen. Figure 1a shows the $200-\mathrm{hPa}$ heights and ageostrophic winds. The ageostrophic wind pattern is somewhat similar to that in Chen's Fig. 12a, particularly in the northwestern quadrant of the panel, although magnitudes can be compared only along cross sections because Chen does not provide a vector scale in Fig. 12. However, the mere presence of ageostrophic wind does not imply a vertical circulation, since part or all of the ageostrophic wind may be nondivergent. Following the ideas of Keyser et al. (1989) and Loughe et al. (1995), we now examine the nondivergent and irrotational components of the ageostrophic wind (Figs. 1b,c). The nondivergent ageostrophic wind generally is much larger in the vicinity of the cross section, accounting for the bulk of the cross-section-normal component of the ageostrophic wind. In particular, in the southeast part of the cross section, where the total ageostrophic wind is almost exactly normal to the height contours, the partitioning in Figs. 1b,c illustrates that most of the transverse component of the flow in the entrance region of the jet streak is nondivergent and is therefore not associated with vertical motion.

If the irrotational ageostrophic wind were exclusively associated with parcels accelerating into the jet streak, southerlies would not extend so far north of the entrance region in Fig. 1c. A larger view (not shown) indicates that the irrotational ageostrophic wind is actually associated with a divergence-convergence couplet with a velocity potential minimum over Taiwan and a maximum over Mongolia. This couplet is associated with the vorticity advection pattern of the upper-level trough over China, a forcing mechanism for vertical motion along the mei-yu front not considered by Chen. Along cross-section $\mathrm{AB}$, much of the ageostrophic wind is antiparallel to the geostrophic wind, suggesting that this ageostrophic wind is attributable more to flow curvature 


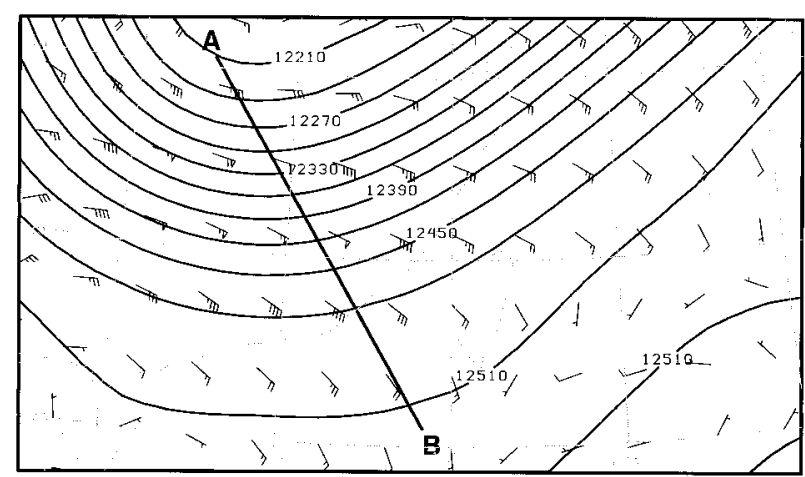

(a) $200 \mathrm{hPa}$ HEIGH $(\mathrm{m})$, PGEO 1200 UTC 23 June 1991

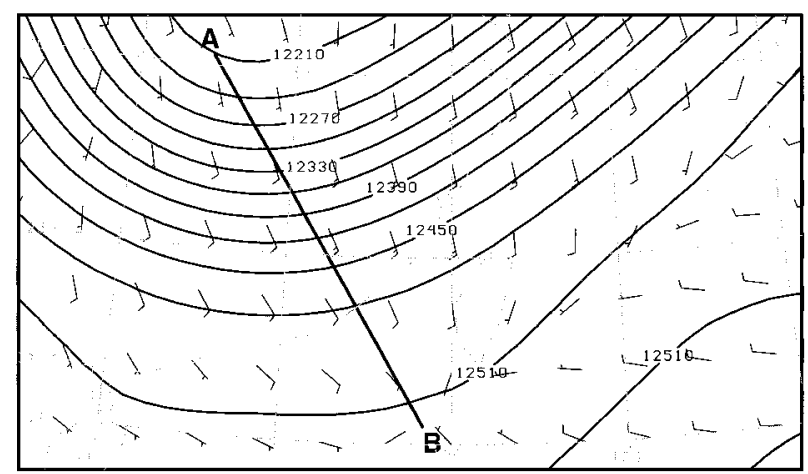

(c) $200 \mathrm{hPa}$ HEIGHT (m), IRROT AGEO 1200 UTC 23 June 1991

effects within the trough than to jet entrance accelerations. In a similar upper-tropospheric flow configuration, the vertical motion associated with the upper-level trough was shown by Cammas and Ramond (1989) to overwhelm the vertical motion signature associated with the attendant jet streaks.

The vertical motion associated with the upper-level ageostrophic wind is so weak that Chen's Fig. 8a shows it to be zero at $6.5 \mathrm{~km}$ prior to the onset of convection. It is not clear how this apparently nonexistent vertical motion could have caused the initial development of the midlevel cloud at $5 \mathrm{~km}$, as claimed by Chen. A comprehensive analysis of the onset of convection should include examination of the possibility that lower or middle tropospheric processes such as moisture advection may have destabilized the atmosphere, allowing convection to break out in the real atmosphere and allowing the convective parameterization to trigger in the model simulation. Once convection is initiated in the model, either through the parameterization or explicitly in Chen's NOCU experiment, the diabatic heating would force vertical motion on its own (Kreitzberg and Perkey 1977). The lack of any upward motion below $6 \mathrm{~km}$ in the adiabatic (NOCLD) simulation (Chen's Fig. 17d) suggests that the convection is essential (and upper-level ageostrophic flow is unimportant) for driving the vertical motion in the lower troposphere.

Chen (p. 357) notes a strong "correlation" between

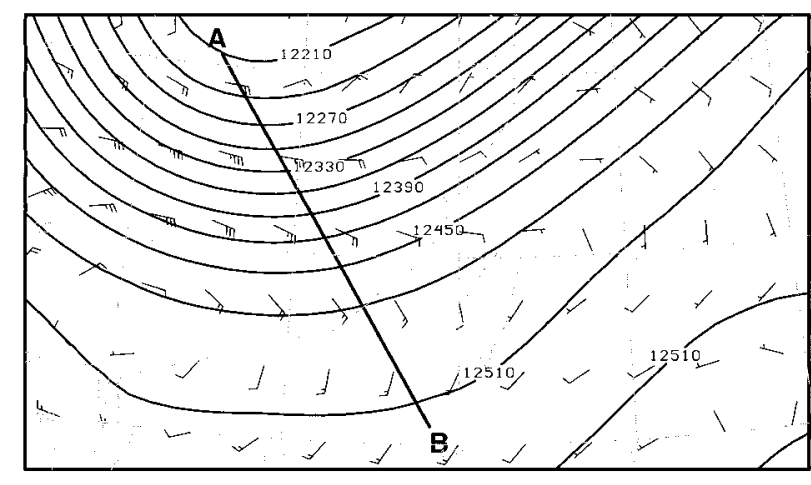

(b) $200 \mathrm{hPa}$ HEIGHT (m), NONDIV AgEo 1200 UTC 23 JUne 1991

FIG. 1. Height $(\mathrm{m})$ and ageostrophic wind (long barb $=5 \mathrm{~m} \mathrm{~s}^{-1}$ ), 200 hPa, 1200 UTC 23 June 1991. (a) Full ageostrophic wind. (b) Nondivergent ageostrophic wind. (c) Irrotational ageostrophic wind. The location of cross section $\mathrm{AB}$ is indicated in each panel.

upper-level divergence and rainfall (Chen's Figs. 5 and 12). This correlation is to be expected; divergence will always be found above deep convection and can easily overwhelm any divergence pattern associated with large-scale or mesoscale "forcing."

\section{Direct circulation forced by convection}

According to Chen's interpretation (pp. 350, 360), the LLJ is produced by the Coriolis deflection of the mesoscale inflow, which is the lower branch of a direct circulation associated with the convection. This is a viable mechanism, although it is perhaps simpler to think of the LLJ as being the nearly balanced response to lowlevel pressure falls produced by convection. [The alternative interpretation of the low-level pressure minima being produced by vorticity convergence (p. 364) is difficult to interpret in this context.] Curiously, the available figures do not show this process taking place, so the case for this mechanism has not been made. Chen's Fig. 9 cross sections show the ageostrophic wind and normal wind speed. The low-level ageostrophic flow toward the northwest appears unassociated with either the instantaneous normal wind speed or the change in normal wind speed from panel to panel. Only at 1600 UTC is there broad southeasterly ageostrophic flow at the appropriate location. In general, there is no consistent mesoinflow at the level of the LLJ core. 
Chen identifies a complete circulation cell driven by convection, with rising motion to the north and sinking motion to the south (Fig. 13), referred to as a "reversed Hadley" circulation. The mesoscale inflow is supposedly the southerly branch of this circulation cell, and a latent-heat-driven circulation is suggested by the comparison between the full and dry simulations (Fig. 19), but we find little evidence for a true closed circulation in the full simulation. Chen presents cross sections at four times in Fig. 8. At 1200 UTC 23 June 1991, since the convection has not yet developed, there is no convectively driven circulation. At 1600 UTC, there is strong upward motion within the cloudy area, but within and immediately to the southeast of this updraft (vector columns 4-6 from the right edge in Fig. 8b), the ageostrophic wind is almost exclusively southeasterly at all levels. In other words, there is no return branch aloft, and therefore no convectively driven circulation. Half a circulation cell appears farther to the southeast, but it occurs entirely within the dry air and appears unrelated to the convection at hand. At 2400 UTC a similar problem exists, with no upper-level northwesterlies present to compose the return branch of the circulation. Only at 2000 UTC does the updraft within the convection appear to be part of a reversed Hadley circulation. While there may be sinking motion beyond the margins of the cross section at other times (Chen, p. 357), it is not part of a circulation cell if it is not fed by air emanating from the updraft. The application of circulation partitioning following Keyser et al. (1989) to Chen's nested simulation may clarify some of these issues.

Given the lack of a consistent closed circulation with low-level southeasterlies and upper-level northwesterlies, it is inappropriate to present a schematic diagram (Chen's Fig. 13) that features a complete circulation cell to the south of the convection and contiguous with the updraft. Were such a cell to exist, however, it would be a direct circulation, not an indirect one as indicated in Fig. 13, since potential temperatures within the updraft are warmer than potential temperatures within the subsiding air according to Chen's Fig. 6a.

\section{Slantwise convection}

Chen (p. 357) describes a curious cause and effect with regard to a slantwise updraft which develops between 1 and 7 km (see Chen's Fig. 8): "a low- to midlevel flow with a sloped structure develops to the left of the vertical convection. This flow induces a weak circulation with a return branch from northern latitudes that provides convergence into the region near the surface underneath the vertical convection." This statement seems to imply that a weak circulation, present because of continuity requirements to provide a source of air for the sloping updraft, is also somehow helping to reinforce the vertical convection. This does not make sense unless the sloping downdraft is evaporatively cooled and can

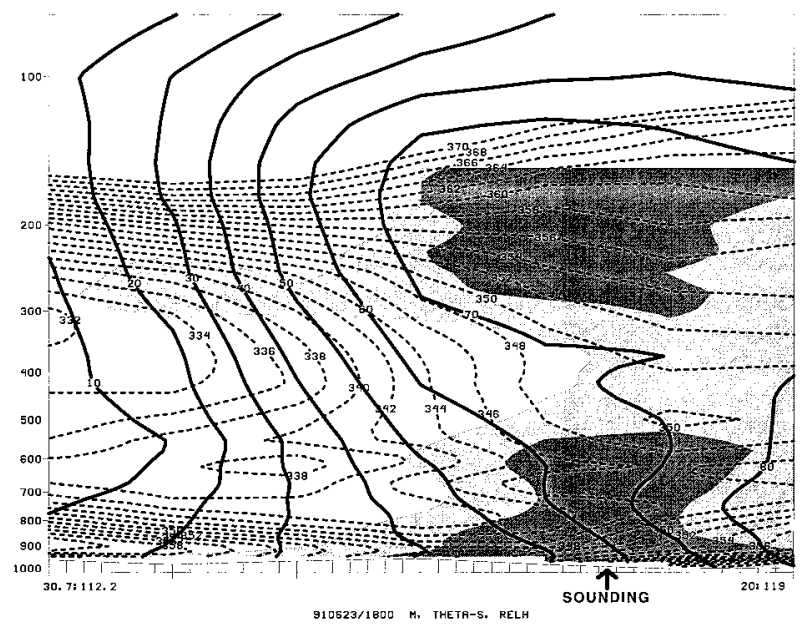

FIG. 2. Cross section of $M$ (solid contours, $\mathrm{m} \mathrm{s}^{-1}$ ), $\theta_{e}^{*}$ (dashed contours, K), and relative humidity (light shading $>70 \%$, dark shading $>90 \%$, along line $\mathrm{AB}$ in Fig. 1.

thereby serve as an outflow boundary to force additional ascent.

To determine the cause of this sloping ascent, Chen uses cross sections of absolute momentum ( $M$ surfaces) and equivalent potential temperature $\left(\theta_{e}\right)$ (Chen's Fig. 21). The figure has been computed incorrectly, however, and even if it were correct it would not be useful for diagnosing symmetric instability.

Chen defines $M=f x+v$, where " $x$ is the distance from the left boundary of the cross section, and $v$ is the wind normal to the cross section" (p. 369). This is a valid definition, notwithstanding the differences of opinion in the literature over whether $M$ or $M_{g}$ (computed using the geostrophic wind) is the more appropriate quantity (Xu 1992; Schultz and Schumacher 1999). However, $M$, as shown in Fig. 21, appears to have been computed using the east-west distance for $x$ rather than the distance along the cross-section axis. Consequently, $M$ along the southeast edge of the cross section is only about half as large as it should be and Chen's analysis of symmetric instability is incorrect. Chen's $M$ surfaces should have looked something like those in Fig. 2, which was created from the NCEP-NCAR reanalysis data.

Chen (p. 369) determines that low-level inflow from the southeast is favored because air parcels can reach the base of the updraft by following $M$ surfaces from the southeast, while they must cross many $M$ surfaces if they approach from the northwest. However, this reasoning cannot be applied after the formation of the lowlevel inflow because the cross-front flow will advect $M$. If the inflow is from the southeast, the horizontal gradient of $M$ will decrease to the southeast, while $M$ surfaces will pile up at the north edge of the updraft. Since the inhomogeneous distribution of $M$ is a natural consequence of the asymmetric inflow, it cannot be invoked as a cause. Prior to the formation of the updraft (Chen's 
Fig. 21a), the cross-front gradient of $M$ is close to uniform, and parcels entering the cross section from the southeast would be unable to follow an $M$ surface to the future location of the updraft. The southeasterly inflow may simply be favored because the updraft is along a frontal zone; the low-level air is more unstable to the southeast than to the northwest.

At upper levels, the inertial instability Chen notes is greatly exaggerated by the error in computing $M$, but some potential for inertial instability is implied even in the cross section from reanalysis data (Fig. 2), wherever $M$ decreases to the right. However, even that instability is exaggerated, because the $M$-based necessary condition for inertial instability assumes two-dimensionality. In reality, the flow is cyclonically curved almost everywhere, and the full absolute vorticity (not shown) is above zero everywhere within the cross section. Further investigation reveals that nearby areas not bisected by the cross section do possess negative absolute vorticity, and might therefore be inertially unstable. We do not know whether Chen's simulations possessed any regions of negative absolute vorticity, although it is true that weak inertial stability could also account for an asymmetric enhancement of the southern branch of the outflow.

It is not immmediately clear how Chen diagnoses conditional symmetric instability (CSI), but it appears that CSI is being inferred by displacing parcels along $M$ surfaces and determining whether they would move into a lower $\theta_{e}$ environment. This approach is valid where the air is saturated, although to assess CSI throughout the domain, saturation equivalent potential temperature $\left(\theta_{e}^{*}\right)$ should be used (Schultz and Schumacher 1999). Furthermore, Chen neglects potential upright instability. Throughout Fig. 21, the low-level high $\theta_{e}$ air is overlain by low $\theta_{e}$ air, so all the air that Chen identifies as being conditionally (more properly, potentially) symmetrically unstable is also potentially unstable to upright convection. As the air mass is lifted vertically and becomes saturated, upright convection would dominate, and slantwise convection may never develop (Bennetts and Sharp 1982).

A similar situation is present in the reanalysis data. In Fig. 2, a shallow layer of saturated high $\theta_{e}^{*}$ air is present near the ground. True, if this air is displaced along an $M$ surface it would be unstable, but the same is true for any vertical displacement. The large amount of upright instability inherent in the atmosphere is shown in Fig. 3, a sounding constructed from gridded data at the point indicated in Fig. 2. Any diagnosis of symmetric instability fails to get to the heart of the matter; if the analysis is correct, deep upright convection is about to develop.

There are, however, regions of Fig. 2 in which, according to parcel theory, CSI is present but conditional upright instability is absent. Assuming two-dimensionality, these locations can be diagnosed by comparing the $M$ and $\theta_{e}^{*}$ contours. Where $\theta_{e}^{*}$ decreases with height,

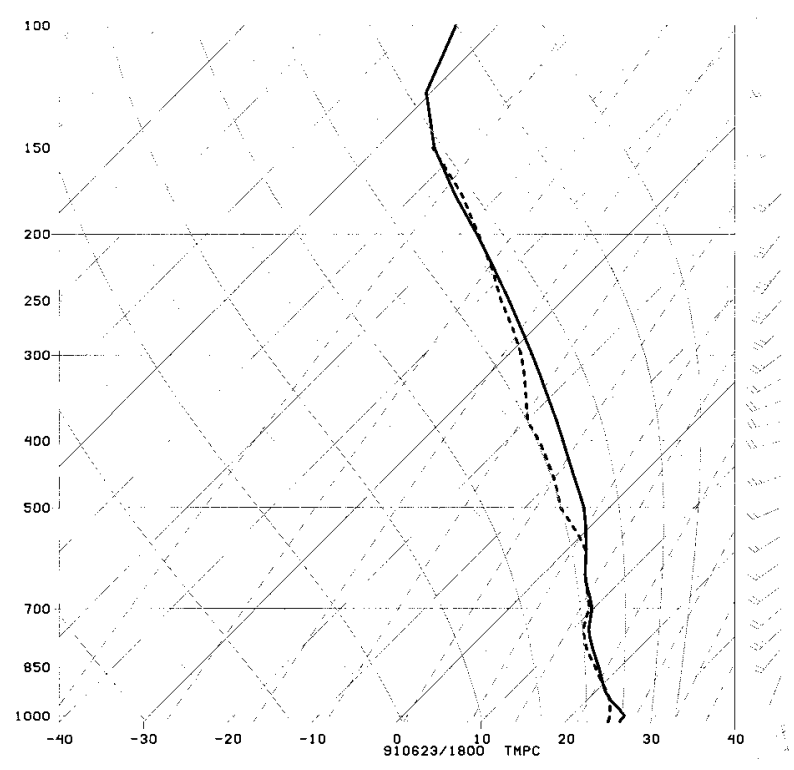

FIG. 3. Gridpoint skew $T$ sounding diagram of temperature (solid) and dew point (dashed) at location indicated by arrow in Fig. 2.

conditional upright instability is present, so upright convection would prevail if the air became saturated. This is the situation for any air parcels originating near the boundary layer. Where $\theta_{e}^{*}$ increases with height, but the $\theta_{e}^{*}$ contours are more vertical than the $M$ surfaces, CSI alone is present. If the air is near saturation there, release of CSI is a real possibility. Such an area is found, for example, along the $M=60 \mathrm{~m} \mathrm{~s}^{-1}$ contour. At $575 \mathrm{hPa}$, $\theta_{e}^{*}$ is $346 \mathrm{~K}$ and the air is nearly saturated. Assuming saturation, this air would be stable to vertical displacements, since $\theta_{e}^{*}$ everywhere above is greater than 346 $\mathrm{K}$. But along the $M$ surface, $\theta_{e}^{*}$ decreases upward, reaching a minimum of $343 \mathrm{~K}$. According to parcel theory (Emanuel 1983), the slantwise unstable parcel would continue ascending until the environmental $\theta_{e}^{*}$ again becomes $346 \mathrm{~K}$, at a level of $250 \mathrm{hPa}$.

CSI is therefore indicated in the reanalysis data, but primarily above $575 \mathrm{hPa}$, well above the level of the slantwise updraft in Chen's simulation. Since Chen does not show the full two-dimensional distribution of $\theta_{e}$ or $\theta_{e}^{*}$ in Fig. 21, we cannot assess the degree of symmetric instability in the simulation. An appropriate analysis of CSI using Chen's simulation would shed light on the nature of the modeled slantwise convection.

\section{Other minor errors}

The sounding shown in Chen's Fig. 7 is described as possessing a boundary layer capping inversion at 850 $\mathrm{hPa}$ (p. 356). This feature is neither an inversion (temperature everywhere decreases with height) nor capping (air from the lowest model layer is uninhibited by the $850 \mathrm{hPa}$ feature and would be unstable all the way to $200 \mathrm{hPa}$ ). 
Several figure captions are erroneous. In Figs. 1 and 2 , the isotherms are identified as being in units of kelvins; they are in Celsius. The scaling factor for divergence is not given for Fig. 12. Figure 14d is labeled with the incorrect time. In Fig. 18, the caption reverses the fields: the pressure is contoured and the wind speed is shaded.

Finally, Chen (pp. 365-367) attempts to paraphrase Hoskins et al. (1978) by stating that geostrophic motions tend to destroy thermal wind balance, that the atmosphere uses its ability to produce ageostrophic wind to restore thermal wind balance, and that the Coriolis deflection of the newly created ageostrophic wind restores the thermal wind balance. This description neglects the equally important role of vertical motions, which act to restore balance beneath jet entrance regions by weakening the temperature gradient.

\section{Summary}

Chen et al. (1998) have clearly shown that the precipitation along the mei-yu front was directly related to the formation of a particular LLJ. We expect these results to apply to many LLJs associated with mei-yu fronts. However, more rigorous diagnosis of Chen's model results are needed before the role and interactions of the upper-level-jet, the convective inflow, and the slantwise ascent can be known.

Acknowledgments. The authors wish to thank Chuck Doswell, Jack Kain, David Stensrud, Edward Zipser, Chris Snyder, Yi-Leng Chen, and an anonymous re- viewer for their helpful comments. J. Nielsen-Gammon was supported by the National Science Foundation under Grant ATM-9553284

\section{REFERENCES}

Bennetts, D. A., and J. C. Sharp, 1982: The relevance of conditional symmetric instability to the prediction of mesoscale frontal rainbands. Quart. J. Roy. Meteor. Soc., 108, 595-602.

Cammas, J.-P., and D. Ramond, 1989: Analysis and diagnosis of the composition of ageostrophic circulations in jet-front systems. Mon. Wea. Rev., 117, 2447-2462.

Chen, C., W.-K. Tao, P.-L. Lin, G. S. Lai, S.-F. Tseng, and T.-C. Chen Wang, 1998: The intensification of the low-level jet during the development of mesoscale convective systems on a mei-yu front. Mon. Wea. Rev., 126, 349-371.

Emanuel, K. A., 1983: The Lagrangian parcel dynamics of moist symmetric instability. J. Atmos. Sci., 40, 2368-2376.

Hoskins, B. J., I. Draghici, and H. C. Davies, 1978: A new look at the w-equation. Quart. J. Roy. Meteor. Soc., 104, 31-38.

Kalnay, E., and Coauthors, 1996: The NCEP/NCAR 40-Year Reanalysis Project. Bull. Amer. Meteor. Soc., 77, 437-471.

Keyser, D., B. D. Schmidt, and D. G. Duffy, 1989: A technique for representing three-dimensional vertical circulations in baroclinic disturbances. Mon. Wea. Rev., 117, 2463-2494.

Kreitzberg, C. W., and D. J. Perkey, 1977: Release of potential instability: Part II: The mechanism of convective/mesoscale interaction. J. Atmos. Sci., 34, 1569-1595.

Loughe, A. F., C.-C. Lai, and D. Keyser, 1995: A technique for diagnosing three-dimensional ageostrophic circulations in baroclinic disturbances on limited-area domains. Mon. Wea. Rev., 123, 1476-1504.

Schultz, D. M., and P. N. Schumacher, 1999: The use and misuse of conditional symmetric instability. Mon. Wea. Rev., in press.

$\mathrm{Xu}, \mathrm{Q}$., 1992: Formation and evolution of frontal rainbands and geostrophic potential vorticity anomalies. J. Atmos. Sci., 49, 629648 . 